\title{
Targeting tumour vasculature as a cancer treatment
}

\author{
CHRISTOPHER A. HONSTVET $\dagger$ and PAMELA F. JONES $\ddagger *$
}

$†$ School of Medicine, University of Leeds, Worsley Building, Clarendon Way, Leeds LS2 9NL, UK

$\Varangle$ Leeds Institute of Molecular Medicine, St James’s University Hospital, Wellcome Trust Brenner Building, Leeds LS9 7TF, UK

(Received 21 February 2007; in final form 22 February 2007)

\begin{abstract}
Modelling blood flow and capillary growth in tumours has been the focus of several research groups with the aim of generating theoretical models that can be used to predict biological behaviour within these systems. Since dysfunctional angiogenesis is seen in a wide range of pathological conditions ranging from cardiovascular, to arthritis, to diabetes, it is easy to see how these models may have a far-reaching influence on future therapeutic strategies.

One major area of anti-cancer treatment is the targeting of the tumour blood vessels. Paradoxically, different approaches taken in angiogenic therapy have different aims. Prevention of new vessel growth, or disruption of existing vessels, are both aimed at preventing sufficient oxygen and nutrients to reach the tumour. However, since drug delivery to tumours is often very poor, due to the disorganised architecture of the tumour vessels, one suggested strategy is to improve circulation within a tumour through "normalisation" of the vasculature which results in more effective anti-tumour drug delivery.

There are currently no theoretical models of vessel regression or vascular disruption even though both are actively targeted by tumour therapies. This article will give an overview of the different mechanisms targeted by different anti-angiogenic strategies and highlight areas that may benefit from the development of predictive theoretical models.
\end{abstract}

Keywords: Angiogenesis; Anti-angiogenesis; Vessel regression; Vascular disrupting agents; Mathematical modelling

\section{Introduction}

Cancer is a leading cause of death worldwide and it is solid tumours that account for over $85 \%$ of cancer mortality [1]. For solid tumours to grow and metastasise they must be supplied with oxygen and nutrients as well as having an efficient means to remove waste products, and this is achieved through the growth of new blood vessels into the tumour [2]. The development of new blood vessels from an existing vasculature is termed angiogenesis and is normally a highly regulated process. Blood vessels have a continual lining with vascular endothelial cells which in the adult are normally kept quiescent though a controlled balance between pro-angiogenic and anti-angiogenic factors (see table 1 and figure 1). However, under certain conditions they can be stimulated to divide through a shift in the balance between these factors, leading to the formation of new vasculature. This angiogenic process has been reviewed elsewhere [3-5] and will not be discussed in detail here.

\footnotetext{
*Corresponding author. Email: p.jones@leeds.ac.uk
} 
Table 1. A few of the known pro-angiogenic factors.

\begin{tabular}{|c|c|}
\hline Pro-angiogenic factor & Mode of action \\
\hline $\begin{array}{l}\text { Vascular endothelial } \\
\text { growth factor (VEGF) }\end{array}$ & $\begin{array}{l}\text { Family of endothelial-specific extracellular ligands that exert robust } \\
\text { angiogenic responses via transmembrane receptor tyrosine } \\
\text { kinases, VEGFRs }\end{array}$ \\
\hline Angiopoietins & $\begin{array}{l}\text { Family of endothelial-specific extracellular ligands that play subtle } \\
\text { roles in the regulation of angiogenesis via the transmembrane } \\
\text { receptor tyrosine kinase. Tie2. Ang-1 acts as maturation/ } \\
\text { stabilisation factor, where its antagonist, Ang2 can exert either } \\
\text { inhibitory or pro-angiogenic effect }\end{array}$ \\
\hline $\begin{array}{l}\text { Fibroblast growth factors: } \\
\text { acidic (aFGF) and basic } \\
\text { (bFGF) }\end{array}$ & $\begin{array}{l}\text { Family of extracellular ligands that exert induce responses via } \\
\text { transmembrane receptor tyrosine kinases, FGFRs. Not specific } \\
\text { for endothelial cells, but can have strong angiogenic effects. } \\
\text { Receptors found widely across many cell types }\end{array}$ \\
\hline $\begin{array}{l}\text { Platelet-derived growth } \\
\text { factors most notably } \\
\text { PDGF-BB }\end{array}$ & $\begin{array}{l}\text { Family of extracellular growth factors that act via specfic } \\
\text { transmembrane receptors, the PDGFRs. Plays a role in pericyte } \\
\text { recruitment during capillary growth and maturation }\end{array}$ \\
\hline $\begin{array}{l}\text { Transforming growth } \\
\text { factor } \beta\end{array}$ & $\begin{array}{l}\text { Extracellular ligand bound by specific transmembrane receptor. Not } \\
\text { specific for endothelial cells but evokes strong angiogenic response }\end{array}$ \\
\hline $\begin{array}{l}\text { Tumour necrosis factor } \\
\text { alpha }(\mathrm{TNF} \alpha)\end{array}$ & $\begin{array}{l}\text { Causes pro-angiogenic response through upregulation of endothelial } \\
\text { adhesion molecules as well as induction of VEGF expression }\end{array}$ \\
\hline
\end{tabular}

There are over 20 factors identified to date, that have been shown to confer some degree of pro-angiogenic activity in vivo or in vitro. Some have activities that are specific to endothelial cells; others have a broader range of target cells.

In adults angiogenesis is normally suppressed but becomes active when a tumour starts to grow. This is known as the angiogenic switch and occurs when a tumour switches to a pro-angiogenic phenotype, through an alteration in the balance between pro- and antiangiogenic factors (figure 1), so inducing the growth of new blood vessels. Factors most commonly increased are vascular endothelial growth factor (VEGF) and basic fibroblast growth factor (bFGF), that can both induce a robust angiogenic response.

Folkman first proposed that this dependency on vasculature renders tumours susceptible to anti-angiogenic targeting more than three decades ago [2]. Since then, much time, effort and

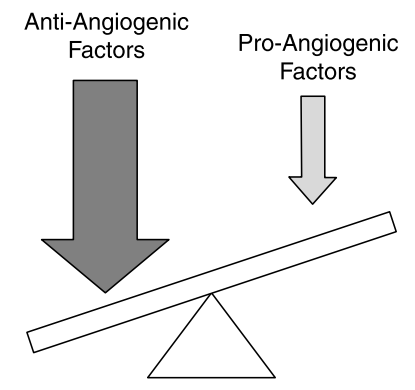

Normal Adult

Angiogenesis kept quiescent

Very little vessel growth

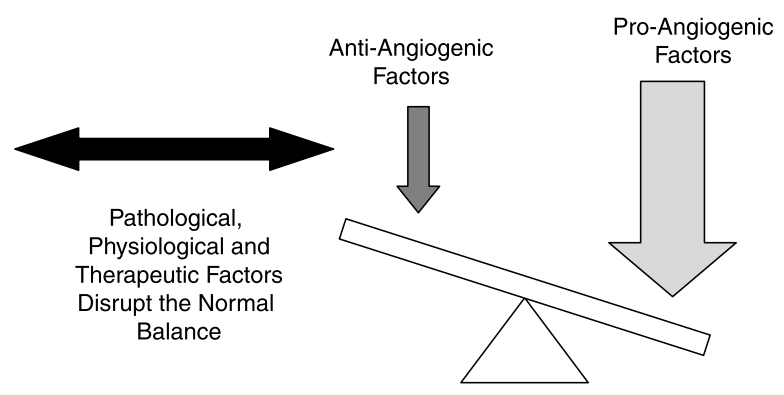

Tumour

New vessels induced

Figure 1. The Angiogenic Balance. In the normal adult, angiogenesis is kept quiescent though the expression of angiogenic inhibitors. In the tumour, the expression of pro-angiogenic factors is increased, while the expression of angiogenic inhibitors is decreased, resulting in a shift towards the pro-angiogenic state. 
Table 2. Some of the drugs currently in development that aim to target the different components of the angiogenesis mechanism.

\begin{tabular}{ll}
\hline Inhibitor & Mode of action \\
\hline $\begin{array}{l}\text { Bevacizumab } \\
\text { Celecoxib }\end{array}$ & $\begin{array}{l}\text { Monoclonal antibody against VEGF. Blocks VEGF binding to receptor } \\
\text { A non-steroidal anti-inflammatory drug (NSAID) that inhibits COX-2, a mediator of VEGF } \\
\text { expression }\end{array}$ \\
$\begin{array}{l}\text { Synthetic inhibitor of matrix metalloproteinases (MMPs) } \\
\text { Thalidomide }\end{array}$ & $\begin{array}{l}\text { Possible COX-2 inhibitor } \\
\text { Angiostatin } \\
\text { Endostatin }\end{array}$ \\
\hline
\end{tabular}

money have been spent on related research and the development of anti-angiogenic therapies, although effective drugs are only now beginning to appear in clinical use. Of the few antiangiogenic drugs that have so far been approved for use, Bevacizumab (also known as Avastin) as a treatment for colorectal cancer is the most effective [6,7] but many others are showing success in phases II and III clinical trials (Cancer Research UK 2007) $\dagger$. In particular angiogenesis inhibitors seem to be useful in stunting the growth of solid tumours before they have become very large and therefore prevent metastasis [8]. However, their action is not suitable for large tumours that have already metastasised. Thus, compounds that directly attack and destroy tumour vasculature vascular disrupting agents (VDAs) are being researched as another possible cancer treatment, and again early indications are showing impressive shutdown of blood supply to tumours resulting in tumour necrosis [9].

It is clear that angiogenesis inhibitors and VDAs will become increasingly prescribed as cancer treatments. Thus, an understanding of their action and the assessment of laboratory and clinical results is important. Also of great interest are possible combination treatment regimes involving both types of vascular-targeted agents with each other or with traditional cancer treatments (chemotherapy and radiotherapy). Having assessed the information, future areas of research and treatment possibilities can be identified.

\section{Anti-angiogenic compounds (angiogenesis inhibitors)}

Tumour angiogenesis is the result of a complex series of interactions between vascular endothelial cells and various growth factors and other naturally occurring chemicals. It has been shown that without neovasculature a tumour cannot expand past a critical mass [10]. Thus, targeting angiogenesis to stop tumour growth and metastasis should be a viable cancer therapy. As angiogenesis is such a multi-step process there are many targets where drugs may exert their effects. Studies have identified a number of possible approaches to targeting antiangiogenic therapies, which are outlined in table 2.

The inhibition of VEGF has shown the most success in the treatment of cancer by targeting angiogenesis. Bevacizumab is the only anti-angiogenic treatment currently available: it is a recombinant humanised monoclonal antibody to VEGF [11] and blocks the VEGF receptor so preventing vascular endothelial cells responding to the angiogenic switch (figure 2). Success in recent trials in colorectal cancer mean the treatment may soon be available for other types of cancer, and results from breast cancer patients show significant increases in 

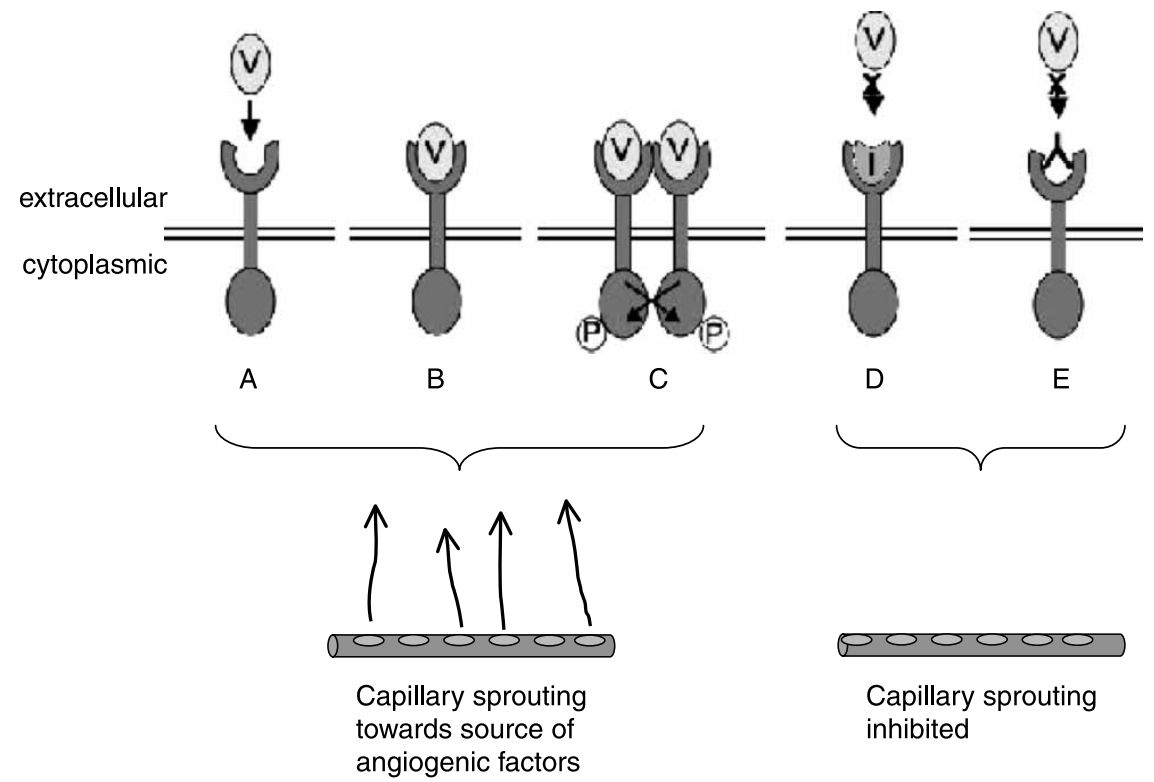

Figure 2. Mode of Action of VEGF Inhibitors VEGF, a soluble extracellular ligand, (A) binds to a specific transmembrane receptor tyrosine kinase (B). Bound VEGFR then forms a dimer with another ligand-bound receptor (C), which brings the two cytoplasmic kinase domains close enough to allow cross-phosphorylation (autophosphorylation) and hence activation of both receptors. This activates the subsequent signalling cascades which results in endothelial cell proliferation, migration and differentiation leading to the formation of new capillaries which sprout towards the angiogenic source (the tumour). Inhibition of VEGF signalling can occur by binding of an inhibitor to the ligand binding site (D), which then prevents VEGF binding to its receptor. Alternatively, binding of an antibody, e.g. Bevacizumab, to an epitope in or close to the ligand binding site (E) prevents VEGF binding. In either case, downstream signalling is not activated and hence angiogenesis is not induced.

progression free survival as well as overall survival [12]. Other trials however, have indicated that Bevacizumab has little therapeutic value for breast cancer in its later stages [13].

Prostaglandins play an important role in angiogenesis. In vivo and in vitro tests have revealed that prostaglandins can induce several growth factors and therefore proliferation of vascular endothelial cells through the stimulation of VEGF [14,15]. Prostaglandin production is regulated by COX-2 and studies have shown COX-2 over-expression is markedly increased in $85-90 \%$ of human colorectal carcinoma [16], as well as in other cancers. Further studies show that patients with elevated COX-2 levels have a poorer prognosis compared to those with low COX-2 levels. Drugs that inhibit COX-2 will therefore inhibit angiogenesis. Thalidomide is an old drug with a poor reputation but recent studies have indicated that it suppresses the LPS-mediated induction of COX-2 [16]. As a result, it has begun to be tested on cancers as a potential anti-angiogenic therapy. So far phase II clinical trial results have been disappointing. In heavily treated breast cancer a $100 \mathrm{mg}$ dose had no therapeutic effect but there were marked increases in the patients quality of life [8]. Ref. [17] also showed that thalidomide had no effects. However, these trials were based on patients with cancer that had already been treated and the treatment had failed. Antiangiogenesis therapy should theoretically be targeted at small tumours before metastasis and so thalidomide is still a potential treatment option.

More recently a novel alternative hypothesis has been presented that predicts that the aim of treating tumours by targeting angiogenesis should be to normalize tumour vasculature as opposed to destroying it. While current anti-angiogenesis therapies can slow the growth 
of tumours they cannot destroy them completely and thus the possibility of remission is high. Jain suggests that tumour vasculature is highly irregular resulting from the angiogenic imbalance (figure 1). These irregular blood vessels follow tortuous routes that do not supply the entire growing tumour. The resultant hypoxia of some regions means that conventional therapies are not effective and the cancer persists. Normalizing the vasculature by restoring the angiogenic balance-possibly through the inhibition of VEGF-may result in more successful delivery and action of conventional cancer treatments [1].

\section{Vascular disrupting agents}

VDAs are low weight molecules that directly attack the blood vessels supplying a tumour resulting in reduction of blood flow and therefore rapid and pronounced tumour necrosis [18]. One of the most important features of VDAs is that they are selective for the tumour-a drug that simply attacked all vessels would be disastrous. Tumour vasculature differs in many ways from normal vasculature and it is this difference that allows VDAs to exert their actions solely on tumours [9]. Table 3 summarises some of these differences and how they might be targeted. The main benefit of VDAs over angiogenesis inhibitors is that they can be effective in fairly advanced cancer where solid tumours are large and there are many sites of metastasis.

Combretastatin A-4 phosphate (CA-4-P) is one of the most promising VDAs. It appears to induce apoptosis in some tumour vascular endothelial cells but the exact mode of action is undefined. It is believed that CA-4-P brings about morphological changes in vessels such as cell retraction, membrane blebbing and the formation of actin stress fibres [9]. Ultimately, all these changes result in an increase in vascular permeability thus inducing ischaemia and interstitial tumour pressure and thus the shutdown of blood supply. The morphological changes can also result in the blocking off of blood vessels and haemorrhagic necrosis of the tumour results [19].

Clinical trials of CA-4-P type drugs are showing promising results. Studies in mice have shown significant tumour growth delays although tumour growth eventually reached normal levels. Further studies with different doses of CA-4-P showed a 100-fold decrease in blood flow to solid tumours with no significant blood flow loss to the brain and other vital organs [9]. Data from early clinical trials are encouraging [20], showing significant reduction in tumour blood flow in 3 out of 4 cases, and in one case even a complete response with the patient remaining

Table 3. Differences between tumour and normal vasculature.

\begin{tabular}{ll}
\hline Features of tumour vasculature & Potential target \\
\hline $\begin{array}{l}\text { Increased vessel tortuosity } \\
\text { Vessels thin walled and fragile } \\
\text { High interstitial pressure within } \\
\text { tumour }\end{array}$ & $\begin{array}{l}\text { Vessels more susceptible to disuption } \\
\text { Easier to disrupt }\end{array}$ \\
$\begin{array}{l}\text { Increased vessel permeability } \\
\text { Massive variability in vascular } \\
\text { density }\end{array}$ & $\begin{array}{l}\text { Easier to deliver drug to tumour tissue } \\
\text { Areas of hypoxia due to Irregular oxygen delivery across } \\
\text { tumour }\end{array}$ \\
$\begin{array}{l}\text { Abnormal endothelial cells- } \\
\text { shape and function }\end{array}$ & $\begin{array}{l}\text { Less mature vessels, easier to disrupt } \\
\text { Possibly surface markers unique to tumour endothelium }\end{array}$ \\
$\begin{array}{l}\text { Tubulin mutations present } \\
\text { Modifications of microtubule } \\
\text { associated protein (MAP) }\end{array}$ & $\begin{array}{l}\text { Targeting disruption of tubulin structures } \\
\text { Specific antigens/less stable structure? }\end{array}$ \\
\hline
\end{tabular}

There are several differences between tumour and normal vasculature that may be potential targets for intervention. 
disease free after 33 months [19]. One drawback of such treatment is that long drug exposure times are required [9]. As a result there are more possible side effects and more chance of normal vasculature being affected. Side effects have included severe tumour pain, dyspnoea and hypertension. However, most side effects have been controlled with minimal medical intervention except where pre existing conditions were worsened, such as hypertension.

The tubulin cytoskeleton of vessels is another target for VDAs. By preventing the proper formation of the cytoskeleton, vessels become more permeable and thus perfuse tumours less effectively. ZD6126 is one such agent that is showing success in laboratory trials and in tumour models [19]. Excitingly, tumour perfusion was reduced by $70 \%$ in just three hours but the vasculature repaired itself in just 6 hours, again indicating that long drug exposure times are required. One explanation of this refers back to the angiogenic switch. Hypoxia seems to induce the pro-angiogenic state. If blood vessels are destroyed rapidly the resulting hypoxia will strongly select for the pro-angiogenic phenotype. By maintaining a dosage of VDAs any new vasculature will be destroyed and thus tumour necrosis will occur. Once the tumour is destroyed there will be no hypoxia and thus no initiation of the pro-angiogenic phenotype.

Another problem encountered when testing VDAs has been a persistent outer ring of tumour left despite continuing treatment [9]. One explanation of this is that outer tumour cells recruit normal vasculature to supply their needs. As the vasculature is normal the doses of VDAs being used do not produce any disruptive effects. The vessels are too organised and too strong to be broken down by drugs aimed at destroying already weakened vessels. Unfortunately metastasis can result from these surrounding tumour cells breaking off and migrating through the blood vessels to other sites. This could provide an explanation as to why VDAs and anti-angiogenic therapies prevent the growth of tumours but fail to prevent the ultimate spread and mortality of cancer.

\section{Combination therapy}

While the theoretical basis behind vascular targeted therapy is exciting, clinical results have been disappointing, with the early trials showing only limited success as a stand-alone treatment for cancer. Conventional therapies (chemotherapy and radiotherapy) also have their limitations, but targeting vasculature as well as the tumour cells themselves may produce a much more toxic environment for cancers and hence combinations of different therapies may have greater success.

Combining more then one anti-angiogenic agent is perhaps one way of improving their effectiveness. There are many regulatory factors involved in angiogenesis and so by targeting several different parts of the mechanism more positive effects may be seen. Recent studies have combined three new angiogenesis inhibitors-marmistat, captopril and fragmin $[19,21]$. One study investigated the effect these agents had on the release of tumour necrosis factor alpha $(\mathrm{TNF} \alpha)$ - a known pro-angiogenic factor on patients with renal cancer. Cancer cells taken from individual patients were stimulated to overproduce TNF $\alpha$ and then treated with the cocktail of angiogenesis inhibitors. The patient response was good with many showing some improvement or a stabilisation of the cancer. The results were linked to lower levels of TNF $\alpha$ in tumour samples [19]. However, this triple combination therapy showed no effect on plasma levels of VEGF [21]. Furthermore, the cancer still persisted in all patients, which still indicates the limited potential of anti-angiogenic therapy as a treatment by itself.

The idea of combining angiogenesis inhibitors with traditional chemotherapy and radiotherapy is the next logical step in the progression of vascular targeted cancer agents. 
The lack of side effects of anti-angiogenic drugs also makes the combination appealing as current therapies have such dramatic side effects (NCI 2005). Bevacizumab has been licensed for use only in combination with chemotherapy and radiotherapy. The failure of the targeted action of chemotherapy and radiotherapy is that tumour cells are able to migrate away from the site of action and escape the treatment along new blood vessels formed by angiogenesis. When VEGF and other pro-angiogenic factors are inhibited new blood vessels are not able to form. Cancer cells are therefore unable to escape from the targeted traditional therapies and so the combination therapy has been shown to have potent anti-tumour effects [22].

Conventional therapies are often limited by the abnormal vasculature of tumours. There are often patches within the tumour that are poorly perfused and only just manage to survive the resulting ischaemia. These areas cannot be targeted by chemotherapy as the drugs have no means of accessing the area. Similarly radiotherapy is less effective as oxygen levels in the tumour are low. By using slightly smaller doses of angiogenesis inhibitors it is possible for the pro-angiogenic state to remain but vessels will be constructed in a more normal way. As a result tumour cells are more efficiently perfused and chemotherapy and radiotherapy are more effective [18].

It is important to look at combination therapy with angiogenesis inhibitors and conventional therapies from a negative angle as well. Chemotherapy is limited by poor blood supply to the tumour and anti-angiogenesis therapy worsens this blood supply. The new drugs are not effective enough to completely remove all vasculature and so some tumour cells remain but with relatively poor blood supply thus preventing chemotherapy or radiotherapy from working. This is especially the case in later stage tumours that are large and have an already fairly established vasculature.

Although some positive clinical trial results have been seen from VDAs the persistent ring of tumour cells that results after treatment prevents it from being an effective cancer treatment. However, by combining VDAs with other therapies the persistent outer ring may be able to be destroyed. The outer ring is supplied by a normal vasculature that supplies it with an efficient blood supply. Thus, chemotherapy agents will be efficiently transported and transfused into the perimeter cancer cells hopefully resulting in tumour necrosis [9]. In this way a complete destruction of solid tumours may be possible.

\section{Conclusions}

Anti-angiogenesis therapy was first proposed in the 1970s and yet few treatments are available to cancer sufferers. Early laboratory work with animals and tumour models showed very exciting results but these have failed to translate in the clinical setting. The question is why. Many of the clinical trials have recruited patients with end stage cancer, which is too late to target effectively new vessel growth. At this stage the vasculature of a tumour is already advanced and the best that angiogenesis inhibitors can hope to achieve is to prevent further growth of the tumour. However, after a while tumour cells seem to continue to grow despite treatment. This could be a result of the complexity of the mechanism of angiogenesis-if one way is blocked perhaps other methods of vessel growth are up-regulated and new vasculature appears. To assess effectively the possible targets of such drugs, trials must be carried out in patients in an early stage of cancer. It is at this stage that theoretically such treatments will have most success. VDAs are a more recent and perhaps more promising development based on a concept of targeting and destroying existing tumour vasculature that is viable for use at all stages of cancer. 
The possibility of developing predictive mathematical/theoretical models of vascular disruption and regression offers an exciting alternative to often long-winded and expensive experimental and clinical protocols. Combination therapies-vascular targeting combined with traditional therapies - need optimising and timing schedules have to be fine-tuned to provide the most effective treatment for the specific cancer. Experimentally, this takes time and resources. The development of theoretical models, which can both reflect pathological cases and predict treatment outcomes, would have a significant impact in this therapeutic area. Such models could be used to predict the most efficacious combinations of specific targeting agents with specific chemotherapy, which would then be used as first choice in validation studies. Similarly, delivery regimes_-schedules as well as site of delivery-could be determined using theoretical models with the most effective being taken further in the clinical setting.

Although mathematical models exist to study capillary sprouting and growth, as well as blood flow across tumours, little, if any, attention has been paid to modelling either regression or disruption of existing blood vessels. Given that clinical diagnosis can only occur when a tumour has progressed well beyond the stage at which a blood supply is needed, targeting existing tumour vasculature is a more viable treatment option than prevention of the initial blood vessel growth. Modelling vascular regression and disruption has the potential to influence treatment strategies, and hence has huge impact on the clinical management of cancer.

\section{References}

[1] Jain, R.K., 2005, Normalization of tumor vasculature: an emerging concept in antiangiogenic therapy, Science, 307, $58-62$.

[2] Folkman, J., 1971, Tumor angiogenesis: therapeutic implications, The New England Journal of Medicine, 285, $1182-1186$.

[3] Risau, W., 1997, Mechanisms of angiogenesis, Nature, 386, 671-674.

[4] Patan, S., 2000, Vasculogenesis and angiogenesis as mechanisms of vascular network formation, growth and remodeling, Journal of Neuro-oncology, 50, 1-15.

[5] Jones, P.F. and Sleeman, B.D., 2006, Angiogenesis-understanding the mathematical challenge, Angiogenesis, 9, 127-138.

[6] Ranieri, G., Patruno, R., Ruggieri, E., Montemurro, S., Valerio, P. and Ribatti, D., 2006, Vascular endothelial growth factor (VEGF) as a target of bevacizumab in cancer: from the biology to the clinic, Current Medicinal Chemistry, 13, 1845-1857.

[7] Thornton, A.D., Ravn, P., Winslet, M. and Chester, K., 2006, Angiogenesis inhibition with bevacizumab and the surgical management of colorectal cancer, The British Journal of Surgery, 93, 1456-1463.

[8] Atiqur Rahman, M. and Toi, M., 2003, Anti-angiogenic therapy in breast cancer, Biomed Pharmacother, 57, 463-470.

[9] Tozer, G.M., Kanthou, C. and Baguley, B.C., 2005, Disrupting tumour blood vessels, Nature Reviews Cancer, $\mathbf{5}, 423-435$

[10] Morabito, A., Sarmiento, R., Bonginelli, P. and Gasparini, G., 2004, Antiangiogenic strategies, compounds, and early clinical results in breast cancer, Critical Reviews in Oncology/Hematology, 49, 91-107.

[11] Ferrara, N., Hillan, K.J. and Novotny, W., 2005, Bevacizumab (Avastin), a humanized anti-VEGF monoclonal antibody for cancer therapy, Biochemical and Biophysical Research Communications, 333, 328-335.

[12] Marx, J., 2005, Encouraging results for second-generation antiangiogenesis drugs, Science, 308, 1248-1249.

[13] Rugo, H.S., 2004, Bevacizumab in the treatment of breast cancer: rationale and current data, Oncologist, 1(1), $43-49$.

[14] Chu, A.J., Chou, T.H. and Chen, B.D., 2004, Prevention of colorectal cancer using COX-2 inhibitors: basic science and clinical applications, Frontiers in Bioscience, 9, 2697-2713.

[15] Gately, S. and Li, W.W., 2004, Multiple roles of COX-2 in tumor angiogenesis: a target for antiangiogenic therapy, Seminars in Oncology, 31, 2-11.

[16] Onn, A., Tseng, J.E. and Herbst, R.S., 2001, Thalidomide, cyclooxygenase-2, and angiogenesis: potential for therapy, Clinical Cancer Research, 7, 3311-3313.

[17] Baidas, S.M., Winer, E.P., Fleming, G.F., Harris, L., Pluda, J.M., Crawford, J.G., Yamauchi, H., Isaacs, C., Hanfelt, J., Tefft, M., Flockhart, D., Johnson, M.D., Hawkins, M.J., Lippman, M.E. and Hayes, D.F., 2000, Phase II evaluation of thalidomide in patients with metastatic breast cancer, Journal of Clinical Oncology, 18, 2710-2717 
[18] Siemann, D.W., Bibby, M.C., Dark, G.G., Dicker, A.P., Eskens, F.A., Horsman, M.R., Marme, D. and Lorusso, P.M., 2005, Differentiation and definition of vascular-targeted therapies, Clinical Cancer Research, 11, 416-420.

[19] Jones, P.H., Christodoulos, K., Dobbs, N., Thavasu, P., Balkwill, F., Blann, A.D., Caine, G.J., Kumar, S., Kakkar, A.J., Gompertz, N., Talbot, D.C., Ganesan, T.S. and Harris, A.L., 2004, Combination antiangiogenesis therapy with marimastat, captopril and fragmin in patients with advanced cancer, British Journal of Cancer, 91, 30-36.

[20] Chaplin, D.J., Horsman, M.R. and Siemann, D.W., 2006, Current development status of small-molecule vascular disrupting agents, Current Opinion in Investigational Drugs, 7, 522-528.

[21] Caine, G.J., Harris, A.L., Christodoulos, K., Lip, G.Y. and Blann, A.D., 2005, Analysis of combination antiangiogenesis therapy on markers of coagulation, platelet activation and angiogenesis in patients with advanced cancer, Cancer Letters, 219, 163-167.

[22] Morabito, A., De Maio, E., Di Maio, M., Normanno, N. and Perrone, F., 2006, Tyrosine kinase inhibitors of vascular endothelial growth factor receptors in clinical trials: current status and future directions, Oncologist, 11, $753-764$. 


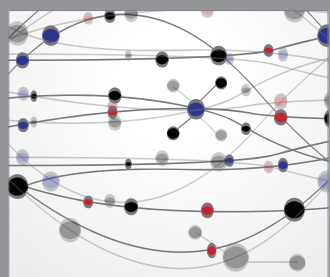

The Scientific World Journal
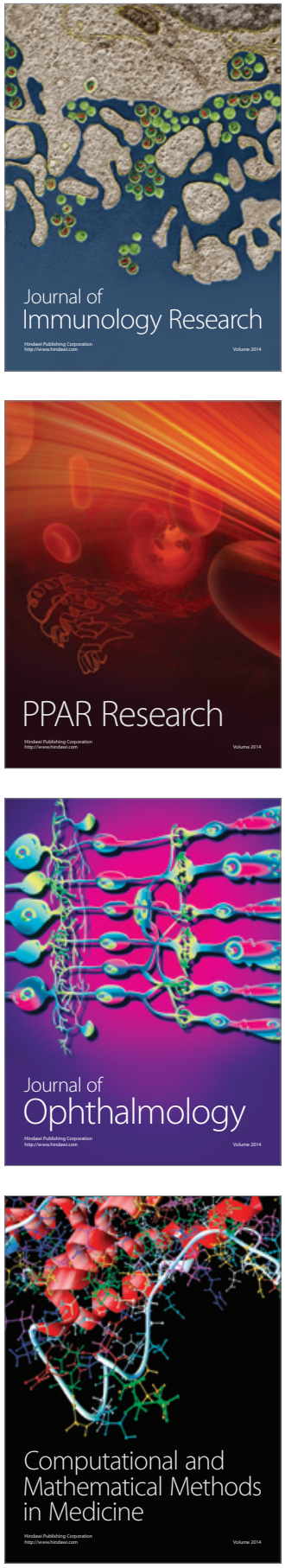

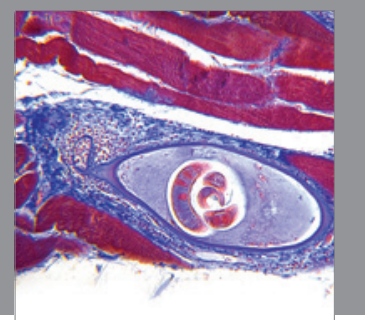

Gastroenterology

Research and Practice
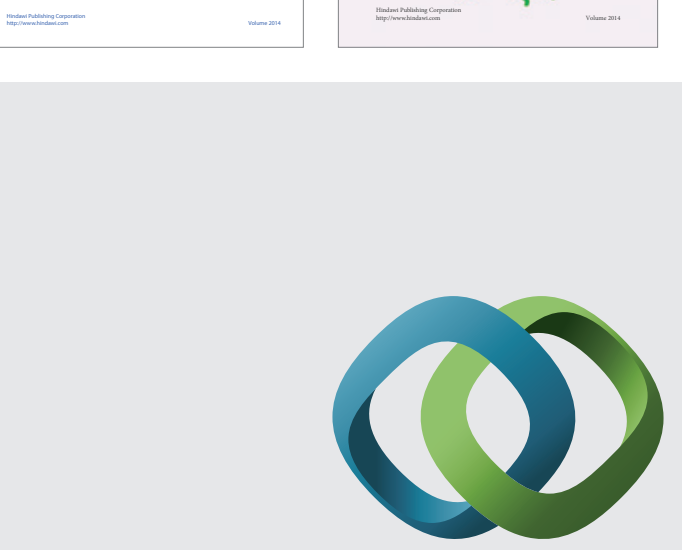

\section{Hindawi}

Submit your manuscripts at

http://www.hindawi.com
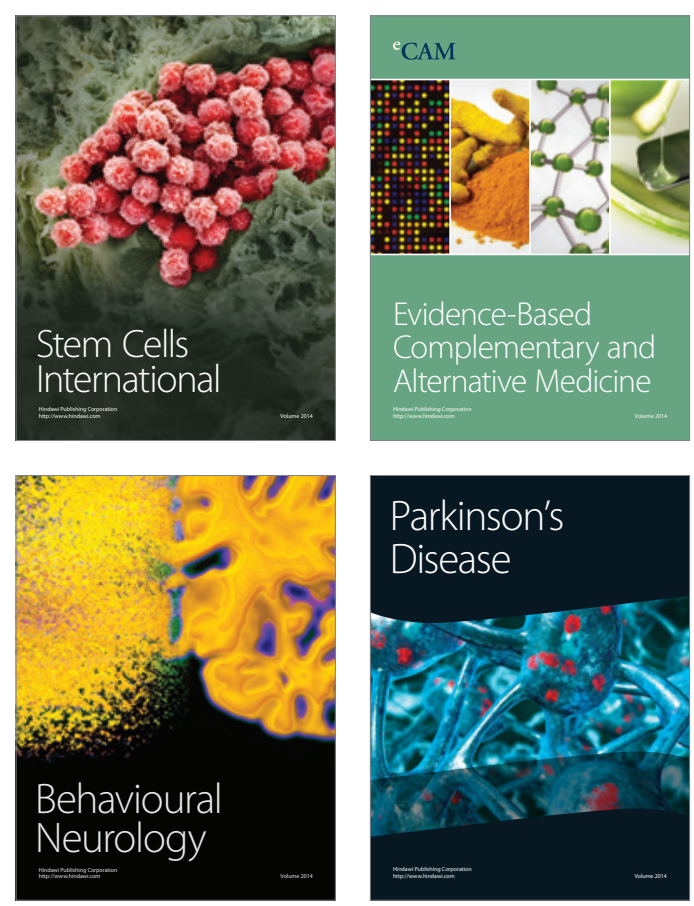

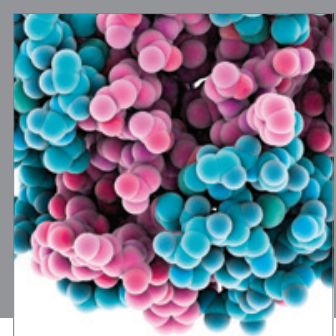

Journal of
Diabetes Research

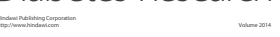

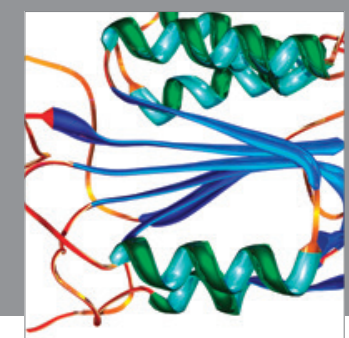

Disease Markers
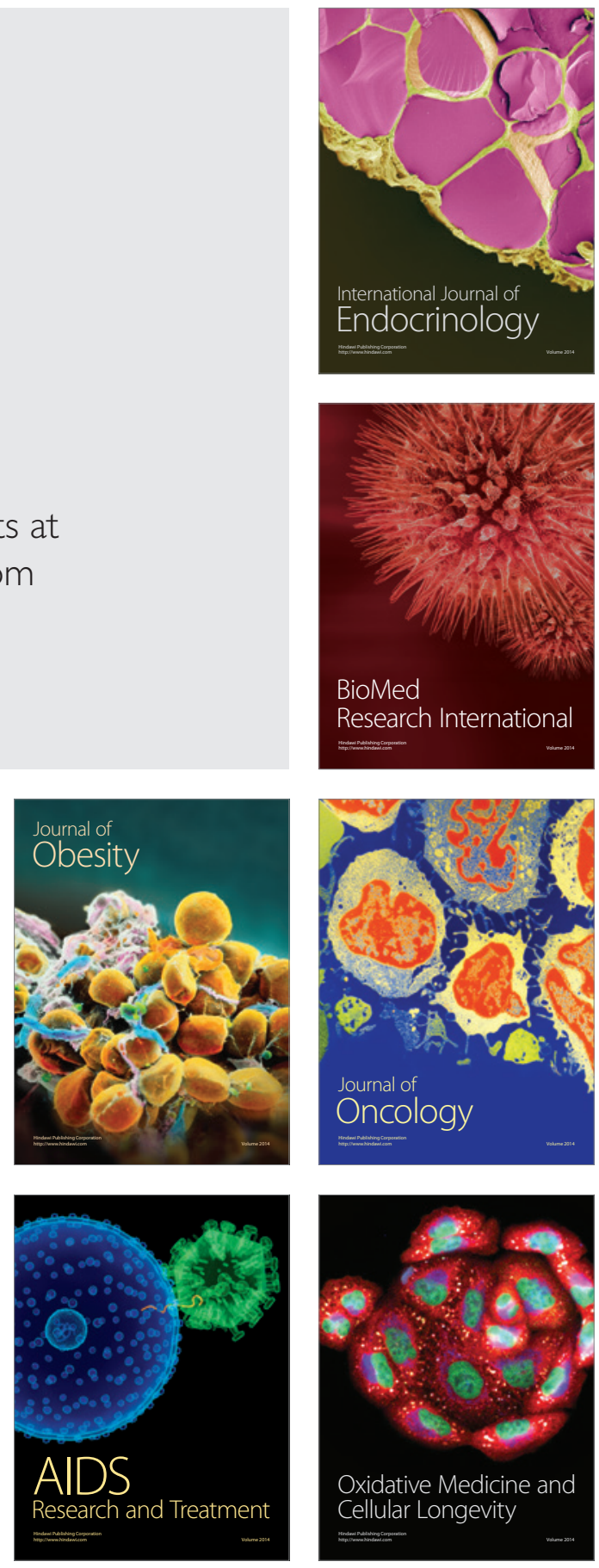